\title{
Optimal Reachability in Divergent Weighted Timed Games ${ }^{\star}$
}

\author{
Damien Busatto-Gaston, Benjamin Monmege and Pierre-Alain Reynier \\ Aix Marseille Univ, LIF, CNRS, France \\ \{damien. busatto, benjamin.monmege, pierre-alain.reynier\}@lif.univ-mrs.fr
}

\begin{abstract}
Weighted timed games are played by two players on a timed automaton equipped with weights: one player wants to minimise the accumulated weight while reaching a target, while the other has an opposite objective. Used in a reactive synthesis perspective, this quantitative extension of timed games allows one to measure the quality of controllers. Weighted timed games are notoriously difficult and quickly undecidable, even when restricted to nonnegative weights. Decidability results exist for subclasses of one-clock games, and for a subclass with non-negative weights defined by a semantical restriction on the weights of cycles. In this work, we introduce the class of divergent weighted timed games as a generalisation of this semantical restriction to arbitrary weights. We show how to compute their optimal value, yielding the first decidable class of weighted timed games with negative weights and an arbitrary number of clocks. In addition, we prove that divergence can be decided in polynomial space. Last, we prove that for untimed games, this restriction yields a class of games for which the value can be computed in polynomial time.
\end{abstract}

\section{Introduction}

Developing programs that verify real-time specifications is notoriously difficult, because such programs must take care of delicate timing issues, and are difficult to debug a posteriori. One research direction to ease the design of real-time software is to automatise the process. We model the situation into a timed game, played by a controller and an antagonistic environment: they act, in a turn-based fashion, over a timed automaton [2, namely a finite automaton equipped with realvalued variables, called clocks, evolving with a uniform rate. A usual objective for the controller is to reach a target. We are thus looking for a strategy of the controller, that is a recipe dictating how to play (timing delays and transitions to follow), so that the target is reached no matter how the environment plays. Reachability timed games are decidable [4], and EXPTIME-complete [20].

If the controller has a winning strategy in a given reachability timed game, several such winning strategies could exist. Weighted extensions of these games have been considered in order to measure the quality of the winning strategy for the controller 91. This means that the game now takes place over a weighted (or priced) timed automaton [5]3], where transitions are equipped with weights, and states with rates of weights (the cost is then proportional to the time spent in this state, with the rate as proportional coefficient). While solving weighted timed automata has been shown to be PSPACE-complete [6] (i.e. the same complexity as the non-weighted version), weighted timed games are known to be undecidable [12. This has led to many restrictions in order to regain decidability, the first and most interesting one being the class of strictly non-Zeno cost with only non-negative weights (in transitions and states) [91]: this hypothesis states that every execution of the timed automaton that follows a cycle of the region automaton has a weight far from 0 (in interval $[1,+\infty)$, for instance).

Less is known for weighted timed games in the presence of negative weights in transitions and/or states. In particular, no results exist so far for a class that does not restrict the number of clocks of the timed automaton to 1 . However, negative weights are particularly interesting from a modelling perspective, for instance in case weights represent the consumption level of a resource (money, energy...) with the possibility to spend and gain some resource. In this work, we introduce a generalisation of the strictly non-Zeno cost hypothesis in the presence of negative weights, that

\footnotetext{
* The first author has been supported by ENS Cachan, Université Paris-Saclay. This work has been funded by the DeLTA project (ANR-16-CE40-0007), and by the SoSI project (PEPS SISC CNRS).
} 
Table 1. Deciding weighted (timed) games with arbitrary weights

\begin{tabular}{|c|c|c|c|}
\cline { 2 - 4 } \multicolumn{1}{c|}{} & Value of a game & Value of a divergent game & Deciding the divergence \\
\hline Untimed & pseudo-poly. [15] & PTIME-complete & NL-complete (unary), PTIME(binary) \\
\hline Timed & Undecidable [12] & 2-EXPTIME, EXPTIME-hard & PSPACE-complete \\
\hline
\end{tabular}

we call divergence. We show the decidability of the class of divergent weighted timed games, with a 2-EXPTIME complexity (and an EXPTIME-hardness lower bound). These complexity results match the ones that could be obtained in the non-negative case from the study of [9]1.

Other types of payoffs than the accumulated weight we study (i.e. total payoff) have been considered for weighted timed games. For instance, energy and mean-payoff timed games have been introduced in [11. They are also undecidable in general. Interestingly, a subclass called robust timed games, not far from our divergence hypothesis, admits decidability results. A weighted timed game is robust if, to say short, every simple cycle (cycle without repetition of a state) has weight non-negative or less than a constant $-\varepsilon$. Solving robust timed game can be done in EXPSPACE, and is EXPTIME-hard. Moreover, deciding if a weighted timed game is robust has complexity 2-EXPSPACE (and coNEXPTIME-hard). In contrast, we show that deciding the divergence of a weighted timed game is a PSPACE-complete problem 11 In terms of modeling power, we do believe that divergence is sufficient for most cases. It has to be noted that extending our techniques and results in the case of robust timed games is intrinsically not possible: indeed, the value problem for this class is undecidable [10].

The property of divergence is also interesting in the absence of time. Indeed, weighted games with reachability objectives have been recently explored as a refinement of mean-payoff games [1415]. A pseudo-polynomial time (i.e. polynomial if weights are encoded in unary) procedure has been proposed to solve them, and they are at least as hard as mean-payoff games. In this article, we also study divergent weighted games, and show that they are the first non-trivial class of weighted games with negative weights solvable in polynomial time. Table 1 summarises our results. We start in Sections 2 and 3 by studying weighted (untimed) games, before considering the timed setting in Sections 4 and 5 .

\section{Weighted games}

We start our study with untimed games. We consider two-player turn-based games played on weighted graphs and denote the two players by Max and Min. A weighted game $2^{2}$ is a tuple $\mathcal{G}=$ $\left\langle V=V_{\text {Min }} \uplus V_{\text {Max }}, V_{t}, A, E\right.$, Weight $\rangle$ where $V$ are vertices, partitioned into vertices belonging to Min $\left(V_{\text {Min }}\right)$ and $\operatorname{Max}\left(V_{\text {Max }}\right), V_{t} \subseteq V_{\text {Min }}$ is a subset of target vertices for player Min, $A$ is an alphabet, $E \subseteq V \times A \times V$ is a set of directed edges, and Weight: $E \rightarrow \mathbb{Z}$ is the weight function, associating an integer weight with each edge. These games need not be finite in general, but in Sections 2 and 3. we limit our study to the resolution of finite weighted games (where all previous sets are finite). We suppose that: $(i)$ the game is deadlock-free, i.e. for each vertex $v \in V$, there is a letter $a \in A$ and a vertex $v^{\prime} \in V$, such that $\left(v, a, v^{\prime}\right) \in E ;(i i)$ the game is deterministic, i.e. for each pair $(v, a) \in V \times A$, there is at most one vertex $v^{\prime} \in V$ such that $\left(v, a, v^{\prime}\right) \in E 3$

A finite play is a finite sequence of edges $\rho=v_{0} \stackrel{a_{0}}{\longrightarrow} v_{1} \stackrel{a_{1}}{\longrightarrow} \cdots \stackrel{a_{k-1}}{\longrightarrow} v_{k}$, i.e. for all $0 \leqslant i<k$, $\left(v_{i}, a_{i}, v_{i+1}\right) \in E$. We denote by $|\rho|$ the length $k$ of $\rho$. We often write $v_{0} \stackrel{\rho}{\rightarrow} v_{k}$ to denote that $\rho$ is a finite play from $v_{0}$ to $v_{k}$. The play $\rho$ is said to be a cycle if $v_{k}=v_{0}$. We let Plays $\mathcal{G}$ be the set of all finite plays in $\mathcal{G}$, whereas Plays $_{\mathcal{G}}^{\text {Min }}$ and Plays ${ }_{\mathcal{G}}^{\text {Max }}$ denote the finite plays that end in a vertex of Min and Max, respectively. A play is then an infinite sequence of consecutive edges.

\footnotetext{
${ }^{1}$ Whereas all divergent weighted game are robust, the converse may not be true, since it is possible to mix positive and negative simple cycles in an SCC.

${ }^{2}$ Weighted games are called min-cost reachability games in 15$]$.

3 Actions are not standardly considered, but they become useful in the timed setting.
} 
A strategy for Min (respectively, Max) is a mapping $\sigma:$ Plays $_{\mathcal{G}}^{\text {Min }} \rightarrow A$ (respectively, $\sigma:$ Plays $_{\mathcal{G}}^{\text {Max }} \rightarrow$ $A$ ) such that for all finite plays $\rho \in \operatorname{Plays}_{\mathcal{G}}^{\text {Min }}$ (respectively, $\rho \in \operatorname{Plays}_{\mathcal{G}}^{\text {Max }}$ ) ending in vertex $v_{k}$, there exists a vertex $v^{\prime} \in V$ such that $\left(v_{k}, \sigma(\rho), v^{\prime}\right) \in E$. A play or finite play $\rho=v_{0} \stackrel{a_{0}}{\longrightarrow} v_{1} \stackrel{a_{1}}{\longrightarrow} \cdots$ conforms to a strategy $\sigma$ of Min (respectively, Max) if for all $k$ such that $v_{k} \in V_{\text {Min }}$ (respectively, $\left.v_{k} \in V_{\text {Max }}\right)$, we have that $a_{k}=\sigma\left(v_{0} \stackrel{a_{0}}{\longrightarrow} v_{1} \cdots v_{k}\right)$. A strategy $\sigma$ is memoryless if for all finite plays $\rho, \rho^{\prime}$ ending in the same vertex, we have that $\sigma(\rho)=\sigma\left(\rho^{\prime}\right)$. For all strategies $\sigma_{\text {Min }}$ and $\sigma_{\text {Max }}$ of players Min and Max, respectively, and for all vertices $v$, we let Play $_{\mathcal{G}}\left(v, \sigma_{\mathrm{Max}}, \sigma_{\mathrm{Min}}\right)$ be the outcome of $\sigma_{\mathrm{Max}}$ and $\sigma_{\mathrm{Min}}$, defined as the unique play conforming to $\sigma_{\mathrm{Max}}$ and $\sigma_{\mathrm{Min}}$ and starting in $v$.

The objective of Min is to reach a target vertex, while minimising the accumulated weight up to the target. Hence, we associate to every finite play $\rho=v_{0} \stackrel{a_{0}}{\longrightarrow} v_{1} \ldots \stackrel{a_{k-1}}{\longrightarrow} v_{k}$ its accumulated weight Weight $_{\mathcal{G}}(\rho)=\sum_{i=0}^{k-1}$ Weight $\left(v_{i}, a_{i}, v_{i+1}\right)$. Then, the weight of an infinite play $\rho=v_{0} \stackrel{a_{0}}{\longrightarrow}$ $v_{1} \stackrel{a_{1}}{\rightarrow} \cdots$, also denoted by Weight $_{\mathcal{G}}(\rho)$, is defined by $+\infty$ if $v_{k} \notin V_{t}$ for all $k \geqslant 0$, or the weight of $v_{0} \stackrel{a_{0}}{\longrightarrow} v_{1} \ldots \stackrel{a_{k-1}}{\longrightarrow} v_{k}$ if $k$ is the first index such that $v_{k} \in V_{t}$. Then, we let $\operatorname{Val}_{\mathcal{G}}\left(v, \sigma_{\mathrm{Min}}\right)$ and $\operatorname{Val}_{\mathcal{G}}\left(v, \sigma_{\text {Max }}\right)$ be the respective values of the strategies:

$$
\begin{aligned}
\operatorname{Val}_{\mathcal{G}}\left(v, \sigma_{\text {Min }}\right) & =\sup _{\sigma_{\text {Max }}} \operatorname{Weight}_{\mathcal{G}}\left(\operatorname{Play}\left(v, \sigma_{\text {Max }}, \sigma_{\text {Min }}\right)\right) \\
\operatorname{Val}_{\mathcal{G}}\left(v, \sigma_{\text {Max }}\right) & =\inf _{\sigma_{\text {Min }}} \operatorname{Weight}_{\mathcal{G}}\left(\operatorname{Play}\left(v, \sigma_{\text {Max }}, \sigma_{\text {Min }}\right)\right) .
\end{aligned}
$$

Finally, for all vertices $v$, we let $\underline{\operatorname{Val}}_{\mathcal{G}}(v)=\sup _{\sigma_{\operatorname{Max}}} \operatorname{Val}_{\mathcal{G}}\left(v, \sigma_{\operatorname{Max}}\right)$ and $\overline{\operatorname{Val}}_{\mathcal{G}}(v)=\inf _{\sigma_{\operatorname{Min}}} \operatorname{Val}_{\mathcal{G}}\left(v, \sigma_{\operatorname{Min}}\right)$ be the lower and upper values of $v$, respectively. We may easily show that $\underline{\mathrm{Val}}_{\mathcal{G}}(v) \leqslant \overline{\mathrm{Val}}_{\mathcal{G}}(v)$ for all $v$. We say that strategies $\sigma_{\text {Min }}^{\star}$ of Min and $\sigma_{\text {Max }}^{\star}$ of Max are optimal if, for all vertices $v$, $\operatorname{Val}_{\mathcal{G}}\left(v, \sigma_{\text {Max }}^{\star}\right)=\underline{\operatorname{Val}}_{\mathcal{G}}(v)$ and $\operatorname{Val}_{\mathcal{G}}\left(v, \sigma_{\text {Min }}^{\star}\right)=\overline{\operatorname{Val}}_{\mathcal{G}}(v)$, respectively. We say that a game $\mathcal{G}$ is determined if for all vertices $v$, its lower and upper values are equal. In that case, we write $\operatorname{Val}_{\mathcal{G}}(v)=\underline{\operatorname{Val}}_{\mathcal{G}}(v)=\overline{\mathrm{Val}}_{\mathcal{G}}(v)$, and refer to it as the value of $v$ in $\mathcal{G}$. Finite weighted games are known to be determined [15]. If the game is clear from the context, we may drop the index $\mathcal{G}$ from all previous notations.

Problems. We want to compute the value of a finite weighted game, as well as optimal strategies for both players, if they exist. The corresponding decision problem, called the value problem, asks whether $\operatorname{Val}_{\mathcal{G}}(v) \leqslant \alpha$, given a finite weighted game $\mathcal{G}$, one of its vertices $v$, and a threshold $\alpha \in \mathbb{Z} \cup\{-\infty,+\infty\}$.

Related work. The value problem is a generalisation of the classical shortest path problem in a weighted graph to the case of two-player games. If weights of edges are all non-negative, a generalised Dijkstra algorithm enables to solve it in polynomial time [21. In the presence of negative weights, a pseudo-polynomial-time (i.e. polynomial with respect to the game where weights are stored in unary) solution has been given in [15], based on a fixed point computation with value iteration techniques. Moreover, the value problem with threshold $-\infty$ is shown to be in NP $\cap$ coNP, and as hard as solving mean-payoff games.

\section{Solving divergent weighted games}

Our first contribution is to solve in polynomial time the value problem, for a subclass of finite weighted games that we call divergent. To the best of our knowledge, this is the first attempt to solve a non-trivial class of weighted games with arbitrary weights in polynomial time. Moreover, the same core technique is used for the decidability result in the timed setting that we will present in the next sections. Let us first define the class of divergent weighted games:

Definition 1. A weighted game $\mathcal{G}$ is divergent when every cycle $\rho$ of $\mathcal{G}$ satisfies Weight $(\rho) \neq 0$.

Divergence is a property of the underlying weighted graph, independent from the repartition of vertices between players. The term divergent reflects that cycling in the game ultimately makes the accumulated weight grow in absolute value. We will first formalise this intuition by analysing the 
strongly connected components (SCC) of the graph structure of a divergent game (the repartition of vertices into players does not matter for the SCC decomposition). Based on this analysis, we will obtain the following results:

Theorem 1. The value problem over finite divergent weighted games is a PTIME-complete problem. Moreover, deciding if a given finite weighted game is divergent is an NL-complete problem when weights are encoded in unary, and PTIME when they are encoded in binary.

SCC analysis. A play $\rho$ in $\mathcal{G}$ is said to be positive (respectively, negative) if Weight $(\rho)>0$ (respectively, Weight $(\rho)<0$ ). It follows that a cycle in a divergent weighted game is either positive or negative. A cycle is said to be simple if no vertices are visited twice (except for the common vertex at the beginning and the end of the cycle). We will rely on the following characterisation of divergent games in terms of SCCs.

Proposition 1. A weighted game $\mathcal{G}$ is divergent if and only if, in each $S C C$ of $\mathcal{G}$, all simple cycles are either all positive, or all negative.

Proof. Let us first suppose that $\mathcal{G}$ is divergent. By contradiction, consider a negative simple cycle $\rho$ (of weight $-p<0$ ) and a positive simple cycle $\rho^{\prime}$ (of weight $p^{\prime}>0$ ) in the same SCC. Let $v$ and $v^{\prime}$ be respectively the first vertices of $\rho$ and $\rho^{\prime}$. By strong connectivity, there exists a finite play $\eta$ from $v$ to $v^{\prime}$ and a finite play $\eta^{\prime}$ from $v^{\prime}$ to $v$. Let us consider the cycle $\rho^{\prime \prime}$ obtained as the concatenation of $\eta$ and $\eta^{\prime}$. If $\rho^{\prime \prime}$ has weight $q>0$, the cycle obtained by concatenating $q$ times $\rho$ and $p$ times $\rho^{\prime \prime}$ has weight 0 , which contradicts the divergence of $\mathcal{G}$. The same reasoning on $\rho^{\prime \prime}$ and $\rho^{\prime}$ proves that $\rho^{\prime \prime}$ can not be negative. Thus, $\rho^{\prime \prime}$ is a cycle of weight 0 , which again contradicts the hypothesis.

Reciprocally, consider a cycle of $\mathcal{G}$. It can be decomposed into simple cycles, all belonging to the same SCC. Therefore they are all positive or all negative. As the accumulated weight of the cycle is the sum of the weights of these simple cycles, $\mathcal{G}$ is divergent.

Computing the values. Consider a divergent weighted game $\mathcal{G}$. Let us start by observing that vertices with value $+\infty$ are those from which Min can not reach the target vertices: thus, they can be computed with the classical attractor algorithm, and we can safely remove them, without changing other values or optimal strategies. In the rest, we therefore assume all values to be in $\mathbb{Z} \cup\{-\infty\}$.

Our computation of the values relies on a value iteration algorithm to find the greatest fixed point of operator $\mathcal{F}:(\mathbb{Z} \cup\{-\infty,+\infty\})^{V} \rightarrow(\mathbb{Z} \cup\{-\infty,+\infty\})^{V}$, defined for every vector $\boldsymbol{x}$ by $\mathcal{F}(\boldsymbol{x})_{v}=0$ if $v \in V_{t}$, and otherwise

$$
\mathcal{F}(\boldsymbol{x})_{v}= \begin{cases}\min _{e=\left(v, a, v^{\prime}\right) \in E} \text { Weight }(e)+\boldsymbol{x}_{v^{\prime}} & \text { if } v \in V_{\text {Min }} \\ \max _{e=\left(v, a, v^{\prime}\right) \in E} \text { Weight }(e)+\boldsymbol{x}_{v^{\prime}} & \text { if } v \in V_{\text {Max }} .\end{cases}
$$

Indeed, this greatest fixed point is known to be the vector of values of the game (see, e.g., [15, Corollary 11]). In [15, it is shown that, by initialising the iterative evaluation of $\mathcal{F}$ with the vector $\boldsymbol{x}^{0}$ mapping all vertices to $+\infty$, the computation terminates after a number of iterations pseudo-polynomial in $\mathcal{G}$ (i.e. polynomial in the number of vertices and the greatest weight in $\mathcal{G}$ ). For $i>0$, we let $\boldsymbol{x}^{i}=\mathcal{F}\left(\boldsymbol{x}^{i-1}\right)$. Notice that the sequence $\left(\boldsymbol{x}^{i}\right)_{i \in \mathbb{N}}$ is non-increasing, since $\mathcal{F}$ is a monotonic operator. Value iteration algorithms usually benefit from decomposing a game into SCCs (in polynomial time), considering them in a bottom-up fashion: starting with target vertices that have value 0, SCCs are then considered in inverse topological order since the values of vertices in an SCC only depend on values of vertices of greater SCCs (in topological order), that have been previously computed.

Example 1. Consider the weighted game of Fig. 1, where Min vertices are drawn with circles, and Max vertices with squares. Vertex $v_{t}$ is the only target. Near each vertex is placed its value. For 


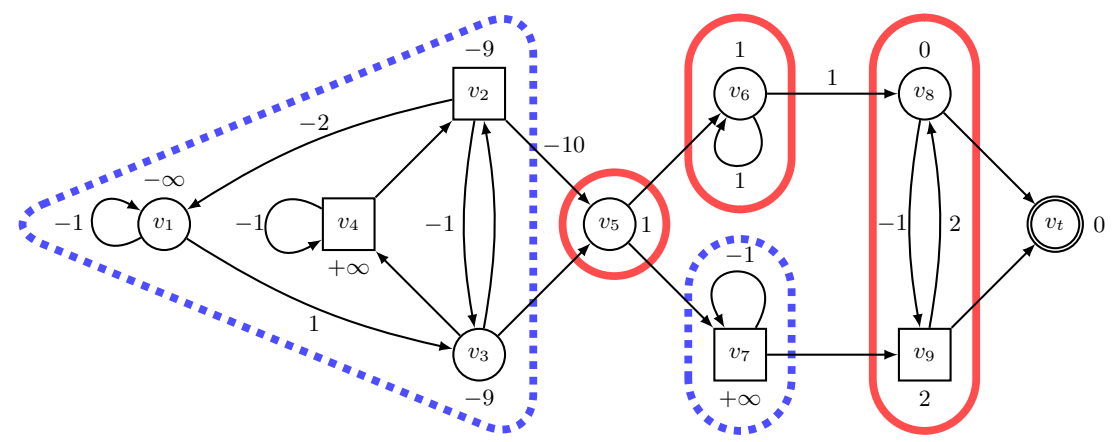

Fig. 1. SCC decomposition of a divergent weighted game: $\left\{v_{1}, v_{2}, v_{3}, v_{4}\right\}$ and $\left\{v_{7}\right\}$ are negative SCCs, $\left\{v_{6}\right\}$ and $\left\{v_{8}, v_{9}\right\}$ are positive SCCs, and $\left\{v_{5}\right\}$ is a trivial positive SCC.

a given vector $\boldsymbol{x}$, we have $\mathcal{F}(\boldsymbol{x})_{v_{8}}=\min \left(0+\boldsymbol{x}_{v_{t}},-1+\boldsymbol{x}_{v_{9}}\right)$ and $\mathcal{F}(\boldsymbol{x})_{v_{2}}=\max \left(-2+\boldsymbol{x}_{v_{1}},-1+\right.$ $\left.\boldsymbol{x}_{v_{3}},-10+\boldsymbol{x}_{v_{5}}\right)$. By a computation of the attractor of $\left\{v_{t}\right\}$ for Min, we obtain directly that $v_{4}$ and $v_{7}$ have value $+\infty$. The inverse topological order on SCCs prescribes then to compute first the values for the $\operatorname{SCC}\left\{v_{8}, v_{9}\right\}$, with target vertex $v_{t}$ associated with value 0 . Then, we continue with SCC $\left\{v_{6}\right\}$, also keeping a new target vertex $v_{8}$ with (already computed) value 0 . For the trivial SCC $\left\{v_{5}\right\}$, a single application of $\mathcal{F}$ suffices to compute the value. Finally, for the SCC $\left\{v_{1}, v_{2}, v_{3}, v_{4}\right\}$, we keep a new target vertex $v_{5}$ with value 14 Notice that this game is divergent, since, in each SCC, all simple cycles have the same sign.

For a divergent game $\mathcal{G}$, Proposition 1 allows us to know in polynomial time if a given SCC is positive or negative, i.e. if all cycles it contains are positive or negative, respectively: it suffices to consider an arbitrary cycle of it, and compute its weight. A trivial SCC (i.e. with a single vertex and no edges) will be arbitrarily considered positive. We now explain how to compute in polynomial time the value of all vertices in a positive or negative SCC.

First, in case of a positive SCC, we show that:

Proposition 2. The value iteration algorithm applied on a positive SCC with $n$ vertices stabilises after at most $n$ steps.

Proof (inspired by techniques used in [9]). Let $W=\max _{e \in E} \mid$ Weight $(e) \mid$ be the greatest weight in the game. There are no negative cycles in the SCC, thus there are no vertices with value $-\infty$ in the SCC, and all values are finite. Let $K$ be an upper bound on the values $\left|\boldsymbol{x}_{v}^{n}\right|$ obtained after $n$ steps of the algorithm 5 Fix an integer $p>(2 K+W(n-1)) n$. We will show that the values obtained after $n+p$ steps are identical to those obtained after $n$ steps only. Therefore, since the algorithm computes non-increasing sequences of values, we have indeed stabilised after $n$ steps only. Assume the existence of a vertex $v$ such that $\boldsymbol{x}_{v}^{n+p}<\boldsymbol{x}_{v}^{n}$. By induction on $p$, we can show (see Lemma 3 in Appendix $\mathrm{A}$ for a detailed proof) the existence of a vertex $v^{\prime}$ and a finite play $\rho$ from $v$ to $v^{\prime}$ with length $p$ and weight $\boldsymbol{x}_{v}^{n+p}-\boldsymbol{x}_{v^{\prime}}^{n}$ : the play is composed of the edges that optimise successively the $\min / \max$ operator in $\mathcal{F}$. This finite play being of length greater than $(2 K+W(n-1)) n$, there is at least one vertex appearing more than $2 K+W(n-1)$ times. Thus, it can be decomposed into at least $2 K+W(n-1)$ cycles and a finite play $\rho^{\prime}$ visiting each vertex at most once. All cycles of the SCC being positive, the weight of $\rho$ is at least $2 K+W(n-1)-(n-1) W=2 K$, bounding from below the weight of $\rho^{\prime}$ by $-(n-1) W$. Then, $\boldsymbol{x}_{v}^{n+p}-\boldsymbol{x}_{v^{\prime}}^{n} \geqslant 2 K$, so $\boldsymbol{x}_{v}^{n+p} \geqslant 2 K+\boldsymbol{x}_{v^{\prime}}^{n} \geqslant K$. But $K \geqslant \boldsymbol{x}_{v}^{n}$, so $\boldsymbol{x}_{v}^{n+p} \geqslant \boldsymbol{x}_{v}^{n}$, and that is a contradiction.

\footnotetext{
${ }^{4}$ This means that, in the definition of $\mathcal{F}$, a vertex $v$ of $V_{t}$ is indeed mapped to its previously computed value, not necessarily 0 .

${ }^{5}$ After $n$ steps, the value iteration algorithm has set to a finite value all vertices, since it extends the attractor computation.
} 
Example 2. For the SCC $\left\{v_{8}, v_{9}\right\}$ of the game in Fig. 1, starting from $\boldsymbol{x}$ mapping $v_{8}$ and $v_{9}$ to $+\infty$, and $v_{t}$ to 0 , after one iteration, $\boldsymbol{x}_{v_{8}}$ changes for value 0 , and after the second iteration, $\boldsymbol{x}_{v_{9}}$ stabilises to value 2 .

Consider then the case of a negative SCC. Contrary to the previous case, we must deal with vertices of value $-\infty$. However, in a negative SCC, those vertices are easy to find 6 . These are all vertices where Max can not unilaterally guarantee to reach a target vertex:

Proposition 3. In a negative SCC with no vertices of value $+\infty$, vertices of value $-\infty$ are all the ones not in the attractor for Max to the targets.

Proof. Consider a vertex $v$ in the attractor for Max to the targets. Then, if Max applies a winning memoryless strategy for the reachability objective to the target vertices, all strategies of Min will generate a play from $v$ reaching a target after at most $|V|$ steps. This implies that $v$ has a finite (lower) value in the game.

Reciprocally, if $v$ is not in the attractor, by determinacy of games with reachability objectives, Min has a (memoryless) strategy $\sigma_{\text {Min }}$ to ensure that no strategy of Max permits to reach a target vertex from $v$. Applying $\sigma_{\text {Min }}$ long enough to generate many negative cycles, before switching to a strategy allowing Min to reach the target (such a strategy exists since no vertex has value $+\infty$ in the game), allows Min to obtain from $v$ a negative weight as small as possible. Thus, $v$ has value $-\infty$.

Thus, we can compute vertices of value $-\infty$ in polynomial time for a negative SCC. Then, finite values of other vertices can be computed in polynomial time with the following procedure. From a negative SCC $\mathcal{G}$ that has no more vertices of value $+\infty$ or $-\infty$, consider the dual (positive) SCC $\widetilde{\mathcal{G}}$ obtained by: $(i)$ switching vertices of Min and Max; (ii) taking the opposite of every weight in edges. Sets of strategies of both players are exchanged in those two games, so that the upper value in $\mathcal{G}$ is equal to the opposite of the lower value in $\widetilde{\mathcal{G}}$, and vice versa. Since weighted games are determined, the value of $\mathcal{G}$ is the opposite of the value of $\widetilde{\mathcal{G}}$. Then, the value of $\mathcal{G}$ can be deduced from the value of $\widetilde{\mathcal{G}}$, for which Proposition 2 applies. We may also interpret this result as follows:

Proposition 4. The value iteration algorithm, initialised with $\boldsymbol{x}_{v}^{0}=-\infty$ (for all $v$ ), applied on a negative SCC with $n$ vertices, and no vertices of value $+\infty$ or $-\infty$, stabilises after at most $n$ steps.

Proof. It is immediate that the vectors computed with this modified value iteration (that computes the smallest fixed point of $\mathcal{F}$ ) are exactly the opposite vectors of the ones computed in the dual positive SCC. The previous explanation is then a justification of the result.

Example 3. Consider the SCC $\left\{v_{1}, v_{2}, v_{3}, v_{4}\right\}$ of the game in Fig. 1 where the value of vertex $v_{5}$ has been previously computed. We already know that $v_{4}$ has value $+\infty$ so we do not consider it further. The attractor of $\left\{v_{5}\right\}$ for Max is $\left\{v_{2}, v_{3}\right\}$, so that the value of $v_{1}$ is $-\infty$. Then, starting from $\boldsymbol{x}_{0}$ mapping $v_{2}$ and $v_{3}$ to $-\infty$, the value iteration algorithm computes this sequence of vectors: $\boldsymbol{x}_{1}=(-9,-\infty)$ (Max tries to maximise the payoff, so he prefers to jump to the target to obtain $-10+1$ than going to $v_{3}$ where he gets $-1-\infty$, while Min chooses $v_{2}$ to still guarantee $\left.0-\infty\right)$, $\boldsymbol{x}_{2}=(-9,-9)$ (now, Min has a choice between the target giving $0+1$ or $v_{3}$ giving $0-9$ ).

The proof for PTIME-hardness comes from a reduction (in logarithmic space) of the problem of solving finite games with reachability objectives [18. To a reachability game, we simply add weights 1 on every transition, making it a divergent weighted game. Then, Min wins the reachability game if and only if the value in the weighted game is lower than $|V|$.

In a divergent weighted game where all values are finite, optimal strategies exist. As observed in [15], Max always has a memoryless optimal strategy, whereas Min may require (finite) memory.

\footnotetext{
${ }^{6}$ This is in contrast with the general case of (non divergent) finite weighted games where the problem of deciding if a vertex has value $-\infty$ is as hard as solving mean-payoff games 15.
} 
Optimal strategies for both players can be obtained by combining optimal strategies in each SCC, the latter being obtained as explained in [15].

Class decision. We explain why deciding the divergence of a weighted game is an NL-complete problem when weighs are encoded in unary. First, to prove the membership in NL, notice that a weighted game is not divergent if and only if there is a positive cycle and a negative cycle, both of length at most $|V|$, and belonging to the same SCC 7 To test this property in NL, we first guess a starting vertex for both cycles. Verifying that those are in the same SCC can be done in NL. Then, we guess the two cycles on-the-fly, keeping in memory their accumulated weights (smaller than $W \times|V|$, with $W$ the biggest weight in the game, and thus of size at most logarithmic in the size of $\mathcal{G}$, if weights are encoded in unary), and stop the on-the-fly exploration when the length of the cycles exceeds $|V|$. Therefore testing divergence is in coNL $=\mathrm{NL}$ [19|25].

The NL-hardness (indeed coNL-hardness, which is equivalent [19]25]) is shown by a reduction of the reachability problem in a finite automaton. More precisely, we consider a finite automaton with a starting state and a different target state without outgoing transitions. We construct from it a weighted game by distributing all states to Min, and equipping all transitions with weight 1 . We also add a loop with weight -1 on the target state and a transition from the target state to the initial state with weight 0 . Then, the game is not divergent if and only if the target can be reached from the initial state in the automaton.

When weights are encoded in binary, the previous decision procedure gives NP membership. However, we can achieve a PTIME upperbound with the following procedure. For every vertex $v$, let $C_{v}=\{\operatorname{Weight}(\rho) \mid \rho$ cycle containing $v\}$. Using Floyd-Warshall's algorithm, it is possible to compute in polynomial time $\inf C_{v}$ (in particular, it detects if $C_{v} \neq \emptyset$ ), as well as $\sup C_{v}$ in a dual fashion. Then, Proposition 1 allows us to guarantee that a weighted game $\mathcal{G}$ is divergent if and only if $0 \notin\left[\inf C_{v}, \sup C_{v}\right]$, for all vertices $v$ such that $C_{v} \neq \emptyset$.

\section{Weighted timed games}

We now turn our attention to a timed extension of the weighted games. We will first define weighted timed games, giving their semantics in terms of infinite weighted games. We let $X$ be a finite set of variables called clocks. A valuation of clocks is a mapping $\nu: X \rightarrow \mathbb{R}_{\geqslant 0}$. For a valuation $\nu$, $d \in \mathbb{R}_{\geqslant 0}$ and $Y \subseteq X$, we define the valuation $\nu+d$ as $(\nu+d)(x)=\nu(x)+d$, for all $x \in X$, and the valuation $\nu[Y \leftarrow 0]$ as $(\nu[Y \leftarrow 0])(x)=0$ if $x \in Y$, and $(\nu[Y \leftarrow 0])(x)=\nu(x)$ otherwise. The valuation $\mathbf{0}$ assigns 0 to every clock. A guard on clocks of $X$ is a conjunction of atomic constraints of the form $x \bowtie c$, where $\bowtie \in\{\leqslant,<,=,>, \geqslant\}$ and $c \in \mathbb{N}$. A valuation $\nu: X \rightarrow \mathbb{R}_{\geqslant 0}$ satisfies an atomic constraint $x \bowtie c$ if $\nu(x) \bowtie c$. The satisfaction relation is extended to all guards $g$ naturally, and denoted by $\nu \models g$. We let $G(X)$ the set of guards over $X$.

A weighted timed game is then a tuple $\mathcal{G}=\left\langle S=S_{\text {Min }} \uplus S_{\text {Max }}, S_{t}, \Delta\right.$, Weight $\rangle$ where $S_{\text {Min }}$ and $S_{\text {Max }}$ are finite disjoint subsets of states belonging to Min and Max, respectively, $S_{t} \subseteq S_{\text {Min }}$ is a subset of target states for player Min, $\Delta \subseteq S \times G(X) \times 2^{X} \times S$ is a finite set of transitions, and Weight: $\Delta \uplus S \rightarrow \mathbb{Z}$ is the weight function, associating an integer weight with each transition and state. Without loss of generality, we may suppose that for each state $s \in S$ and valuation $\nu$, there exists a transition $\left(s, g, Y, s^{\prime}\right) \in \Delta$ such that $\nu \models g$.

The semantics of a weighted timed game $\mathcal{G}$ is defined in terms of the infinite weighted game $\mathcal{H}$ whose vertices are configurations of the weighted timed game. A configuration is a pair $(s, \nu)$ with a state and a valuation of the clocks. Configurations are split into players according to the state. A configuration is final if its state is final. The alphabet of $\mathcal{H}$ is given by $\mathbb{R}_{\geqslant 0} \times \Delta$ and will encode the delay that a player wants to spend in the current state, before firing a certain transition. For every delay $d \in \mathbb{R}_{\geqslant 0}$, transition $\delta=\left(s, g, Y, s^{\prime}\right) \in \Delta$ and valuation $\nu$, there is an

\footnotetext{
${ }^{7}$ If the game is not divergent, there exists an SCC containing a negative simple cycle and a positive one by Proposition 11. This implies the existence of a negative cycle and a positive cycle in the same SCC, both of length at most $|V|$. Reciprocally, this property implies the non-divergence, by the same proof as for Proposition 1
} 
edge $(s, \nu) \stackrel{d, \delta}{\longrightarrow}\left(s^{\prime}, \nu^{\prime}\right)$ if $\nu+d \models g$ and $\nu^{\prime}=(\nu+d)[Y \leftarrow 0]$. The weight of such an edge $e$ is given by $d \times$ Weight $(s)+$ Weight $(\delta)$.

Plays, strategies, and values in the weighted timed game $\mathcal{G}$ are then defined as the ones in $\mathcal{H}$. It is known that weighted timed games are determined $\left(\underline{\mathrm{Val}}_{\mathcal{G}}(s, \nu)=\overline{\mathrm{Val}}_{\mathcal{G}}(s, \nu)\right.$ for all state $s$ and valuation $\nu) 8$

As usual in related work [1910, we assume that all clocks are bounded, i.e. there is a constant $M \in \mathbb{N}$ such that every transition of the weighted timed games is equipped with a guard $g$ such that $\nu \models g$ implies $\nu(x) \leqslant M$ for all clocks $x \in X$. We will rely on the crucial notion of regions, as introduced in the seminal work on timed automata [2]: a region is a set of valuations, that are all time-abstract bisimilar. There is only a finite number of regions and we denote by $\operatorname{Reg}(X, M)$ the set of regions associated with set of clocks $X$ and maximal constant $M$ in guards. For a valuation $\nu$, we denote by $[\nu]$ the region that contains it. A region $r^{\prime}$ is said to be a time successor of region $r$ if there exist $\nu \in r, \nu^{\prime} \in r^{\prime}$, and $d>0$ such that $\nu^{\prime}=\nu+d$. Moreover, for $Y \subseteq X$, we let $r[Y \leftarrow 0]$ be the region where clocks of $Y$ are reset.

The region automaton $\mathcal{R}(\mathcal{G})$ of a game $\mathcal{G}=\left\langle S=S_{\text {Min }} \uplus S_{\text {Max }}, S_{t}, \Delta\right.$, Weight $\rangle$ is the finite automaton with states $S \times \operatorname{Reg}(X, M)$, alphabet $\Delta$, and a transition $(s, r) \stackrel{\delta}{\rightarrow}\left(s^{\prime}, r^{\prime}\right)$ labelled by $\delta=\left(s, g, Y, s^{\prime}\right)$ if there exists a region $r^{\prime \prime}$ time successor of $r$ such that $r^{\prime \prime}$ satisfies the guard $g$, and $r^{\prime}=r^{\prime \prime}[Y \leftarrow 0]$. We call path an execution (not necessarily accepting) of this finite automaton, and we denote by $\pi$ the paths. A play $\rho$ in $\mathcal{G}$ is projected on a execution $\pi$ in $\mathcal{R}(\mathcal{G})$, by replacing actual valuations by the regions containing them: we say that $\rho$ follows path $\pi$. It is important to notice that, even if $\pi$ is a cycle (i.e. starts and ends in the same state of the region automaton), there may exist plays following it in $\mathcal{G}$ that are not cycles, due to the fact that regions are sets of valuations.

Problems. As in weighted (untimed) games, we consider the value problem, mimicked from the one in $\mathcal{H}$. Precisely, given a weighted timed game $\mathcal{G}$, a configuration $(s, \nu)$ and a threshold $\alpha \in$ $\mathbb{Z} \cup\{-\infty,+\infty\}$, we want to know whether $\operatorname{Val}_{\mathcal{G}}(s, \nu) \leqslant \alpha$. In the context of timed games, optimal strategies may not exist. We generally focus on $\varepsilon$-optimal strategies, that guarantee the optimal value, up to a small error $\varepsilon$.

Related work. In the one-player case, computing the optimal value and an $\varepsilon$-optimal strategy for weighted timed automata is known to be PSPACE-complete [6]. In the two-player case, much work for weighted timed games (also called priced timed games in the literature) has been achieved in the case of non-negative weights. In this setting, the value problem is undecidable [1210]. To obtain decidability, one possibility is to limit the number of clocks to 1 : then, there is an exponential-time algorithm to compute the value as well as $\varepsilon$-optimal strategies $7[2317$, whereas the problem is only known to be PTIME-hard. The other possibility to obtain a decidability result [19] is to enforce a semantical property of divergence, originally called strictly non-Zeno cost: it asks that every play following a cycle in the region automaton has weight at least 1.

In the presence of negative weights, undecidability even holds for weighted timed games with only 2 clocks 16 (for the existence problem asking if a strategy of player Min can guarantee a given threshold). Only the 1-clock restriction has been studied so far allowing one to obtain an exponential-time algorithm, under restrictions on the resets of the clock in cycles [13]. For weighted timed games, the strictly non-Zeno cost property has only been defined and studied in the absence of negative weights [9]. As already mentioned in the introduction, the notion is close, but not equivalent, to the one of robust weighted timed games, studied for mean-payoff and energy objectives [11. In the next section, we extend the strictly non-Zeno cost property to negative weights calling it the divergence property, in order to obtain decidability of a large class of multi-clocks weighted timed games in the presence of arbitrary weights.

\footnotetext{
${ }^{8}$ The result is stated in 13 for weighted timed games (called priced timed games) with one clock, but the proof does not use the assumption on the number of clocks.
} 


\section{$5 \quad$ Solving divergent weighted timed games}

We introduce divergent weighted timed games, as an extension of divergent weighted games to the timed setting.

Definition 2. A weighted timed game $\mathcal{G}$ is divergent when every finite play $\rho$ in $\mathcal{G}$ following a cycle in the region automaton $\mathcal{R}(\mathcal{G})$ satisfies Weight $(\rho) \notin(-1,1) \underline{9}$

The weight is not only supposed to be different from 0 , but also far from 0 : otherwise, the original intuition on the ultimate growing of the values of plays would not be fulfilled. If $\mathcal{G}$ has only non-negative weights on states and transitions, this definition matches with the strictly nonZeno cost property of [9, Thm. 6]. Our contributions summarise as follows:

Theorem 2. The value problem over divergent weighted timed games is decidable in 2-EXPTIME, and is EXPTIME-hard. Moreover, deciding if a given weighted timed game is divergent is a PSPACEcomplete problem.

Remember that these complexity results match the ones that can be obtained from the study of [9] for non-negative weights.

SCC analysis. Keeping the terminology of the untimed setting, a cycle $\pi$ of $\mathcal{R}(\mathcal{G})$ is said to be positive (respectively, negative) if every play $\rho$ following $\pi$ satisfies Weight $(\rho) \geqslant 1$ (respectively, Weight $(\rho) \leqslant-1$ ). By definition, every cycle of the region automaton of a divergent weighted timed game is positive or negative. Moreover, notice that checking if a cycle $\pi$ is positive or negative can be done in polynomial time with respect to the length of $\pi$. Indeed, the set $\{$ Weight $(\rho) \mid \rho$ is a play following $\pi\}$ is an interval, as the image of a convex set by an affine function (see [6, Sec. 3.2] for explanation), and the extremal points of this interval can be computed in polynomial time by solving a linear problem [6, Cor. 1]. We first transfer in the timed setting the characterisation of divergent games in terms of SCCs that we relied on in the untimed setting:

Proposition 5. A weighted timed game $\mathcal{G}$ is divergent if and only if, in each $S C C$ of $\mathcal{R}(\mathcal{G})$, simple cycles are either all positive, or all negative.

The proof of the reciprocal follows the exact same reasoning than for weighted games (see Proposition (1). For the direct implication, the situation is more complex: we need to be more careful in the composition of cycles with each others, and weights in the timed game are no longer integers, forbidding the arithmetical reasoning we applied. To help us, we will rely on the cornerpoint abstraction introduced in 8 to study multi-weighted timed automata. It consists in adding a weighted information to the edges $(s, r) \stackrel{\delta}{\rightarrow}\left(s^{\prime}, r^{\prime}\right)$ of the region automaton. Since the weights depend on the exact valuations $\nu$ and $\nu^{\prime}$, taken in regions $r$ and $r^{\prime}$, respectively, the weight of such an edge in the region automaton is computed for each pair of corners of the regions. Formally, corners of region $r$ are valuations in $\bar{r} \cap \mathbb{N}^{X}$ (where $\bar{r}$ denotes the topological closure of $r$ ). Since corners do not necessarily belong to their regions, we must consider a modified version $\overline{\mathcal{G}}$ of the game $\mathcal{G}$ where all strict inequalities of guards have been replaced with non-strict ones. Then, for a path $\pi$ in $\mathcal{R}(\mathcal{G})$, we denote by $\bar{\pi}$ the equivalent of path $\pi$ in $\mathcal{R}(\overline{\mathcal{G}})$.

In the following, our focus is on cycles of the region automaton, so we only need to consider the aggregation of all the behaviours following a cycle. Inspired by the folded orbit graphs (FOG) introduced in [22], we define the folded orbit graph FOG $(\pi)$ of a cycle $\pi=\left(s_{1}, r=r_{1}\right) \stackrel{\delta_{1}}{\longrightarrow}$ $\left(s_{2}, r_{2}\right) \stackrel{\delta_{2}}{\longrightarrow} \cdots \stackrel{\delta_{n}}{\longrightarrow}\left(s_{1}, r\right)$ in $\mathcal{R}(\mathcal{G})$ as a graph whose vertices are corners of region $r$, and that contains an edge from corner $v$ to corner $v^{\prime}$ if there exists a finite play $\bar{\rho}$ in $\overline{\mathcal{G}}$ from $\left(s_{1}, v\right)$ to $\left(s_{1}, v^{\prime}\right)$ following $\bar{\pi}$ jumping from corners to corner 10 . We fix such a finite play $\bar{\rho}$ arbitrarily and label the

\footnotetext{
$\overline{9}$ As in [9], we could replace $(-1,1)$ by $(-\kappa, \kappa)$ to define a notion of $\kappa$-divergence. However, since weights and guard constraints in weighted timed games are integers, for $\kappa \in(0,1)$, a weighted timed game $\mathcal{G}$ is $\kappa$-divergent if and only if it is divergent.

${ }^{10}$ Notice that if there is a play from $\left(s_{1}, v\right)$ to $\left(s_{1}, v^{\prime}\right)$ in $\overline{\mathcal{G}}$, there is another one that only jumps at corners of regions.
} 
edge between $v$ and $v^{\prime}$ in the FOG by this play: it is then denoted by $v \stackrel{\bar{\rho}}{\rightarrow} v^{\prime}$. Moreover, since $\bar{\rho}$ jumps from corners to corners, its weight Weight $(\bar{\rho})$ is an integer, conforming to the definitions of the corner-point abstraction of [8]. Following [8, Prop. 5] (see Appendix B for a complete proof), it is possible to find a play $\rho$ in $\mathcal{G}$ close to $\bar{\rho}$, in the sense that we control the difference between their respective weights:

Lemma 1. For all $\varepsilon>0$ and edge $v \stackrel{\bar{\rho}}{\rightarrow} v^{\prime}$ of $\operatorname{FOG}(\pi)$, there exists a play $\rho$ in $\mathcal{G}$ following $\pi$ such that $\mid$ Weight $(\rho)-$ Weight $(\bar{\rho}) \mid \leqslant \varepsilon$.

In order to prove the direct implication of Proposition 5 , suppose now that $\mathcal{G}$ is divergent, and consider two simple cycles $\pi$ and $\pi^{\prime}$ in the same SCC of $\mathcal{R}(\mathcal{G})$. We need to show that they have the same sign. Lemma 2 will first take care of the case where $\pi$ and $\pi^{\prime}$ share a state $(s, r)$.

Lemma 2. If $\mathcal{G}$ is divergent and two cycles $\pi$ and $\pi^{\prime}$ of $\mathcal{R}(\mathcal{G})$ share a state $(s, r)$, they are either both positive or both negative.

Proof. Suppose by contradiction that $\pi$ is negative and $\pi^{\prime}$ is positive. We assume that $(s, r)$ is the first state of both $\pi$ and $\pi^{\prime}$, possibly performing cyclic permutations of states if necessary. We construct a graph $\operatorname{FOG}\left(\pi, \pi^{\prime}\right)$ as the union of $\mathrm{FOG}(\pi)$ and $\operatorname{FOG}\left(\pi^{\prime}\right)$ (that share the same set of vertices), colouring in blue the edges of $\operatorname{FOG}(\pi)$ and in red the edges of $\mathrm{FOG}\left(\pi^{\prime}\right)$. A path in $\operatorname{FOG}\left(\pi, \pi^{\prime}\right)$ is said blue (respectively, red) when all of its edges are blue (respectively, red).

We assume first that there exists in $\mathrm{FOG}\left(\pi, \pi^{\prime}\right)$ a blue cycle $C$ and a red cycle $C^{\prime}$ with the same first vertex $v$. Let $k$ and $k^{\prime}$ be the respective lengths of $C$ and $C^{\prime}$, so that $C$ can be decomposed as $v \stackrel{\overline{\rho_{1}}}{\longrightarrow} \cdots \stackrel{\overline{\rho_{k}}}{\longrightarrow} v$ and $C^{\prime}$ as $v \stackrel{\overline{\rho_{1}^{\prime}}}{\longrightarrow} \cdots \stackrel{\overline{\rho_{k^{\prime}}^{\prime}}}{\longrightarrow} v$, where $\overline{\rho_{i}}$ are plays following $\bar{\pi}$ and $\overline{\rho_{i}^{\prime}}$ are plays following $\overline{\pi^{\prime}}$, all jumping only on corners of regions. Let $\bar{\rho}$ be the concatenation of $\overline{\rho_{1}}, \ldots, \overline{\rho_{k}}$, and $\overline{\rho^{\prime}}$ be the concatenation of $\overline{\rho_{1}^{\prime}}, \ldots, \overline{\rho_{k^{\prime}}^{\prime}}$. Recall that $w=|\operatorname{Weight}(\bar{\rho})|$ and $w^{\prime}=\left|\operatorname{Weight}\left(\overline{\rho^{\prime}}\right)\right|$ are integers. Since $\pi$ is negative, so is $\pi^{k}$, the concatenation of $k$ copies of $\pi$ (the weight of a play following it is a sum of weights all below -1 ). Therefore, $\bar{\rho}$, that follows $\pi^{k}$, has a weight Weight $(\bar{\rho}) \leqslant-1$. Similarly, Weight $\left(\overline{\rho^{\prime}}\right) \geqslant 1$. We consider the cycle $C^{\prime \prime}$ obtained by concatenating $w^{\prime}$ copies of $C$ and $w$ copies of $C^{\prime}$. Similarly, we let $\overline{\rho^{\prime \prime}}$ be the play obtained by concatenating $w^{\prime}$ copies of $\bar{\rho}$ and $w$ copies of $\overline{\rho^{\prime}}$. By Lemma 1, there exists a play $\rho^{\prime \prime}$ in $\mathcal{G}$, following $C^{\prime \prime}$ such that $\left|\operatorname{Weight}\left(\rho^{\prime \prime}\right)-\operatorname{Weight}\left(\overline{\rho^{\prime \prime}}\right)\right| \leqslant 1 / 3$. But Weight $\left(\overline{\rho^{\prime \prime}}\right)=\operatorname{Weight}(\bar{\rho}) w^{\prime}+\operatorname{Weight}\left(\overline{\rho^{\prime}}\right) w=0$, so Weight $\left(\rho^{\prime \prime}\right) \in(-1,1)$ : this contradicts the divergence of $\mathcal{G}$, since $\rho^{\prime \prime}$ follows the cycle of $\mathcal{R}(\mathcal{G})$ composed of $w^{\prime}$ copies $\pi^{k}$ and $w$ copies of $\pi^{\prime k^{\prime}}$ of $\mathcal{R}(\mathcal{G})$.

We now return to the general case, where $C$ and $C^{\prime}$ may not exist. Since $\operatorname{FOG}(\pi)$ and $\operatorname{FOG}\left(\pi^{\prime}\right)$ are finite graphs with no deadlocks (every corner has an outgoing edge), from every corner of FOG $\left(\pi, \pi^{\prime}\right)$, we can reach a blue simple cycle, as well as a red simple cycle. Since there are only a finite number of simple cycles in $\operatorname{FOG}\left(\pi, \pi^{\prime}\right)$, there exists a blue cycle $C$ and a red cycle $C^{\prime}$ that can reach each other in $\operatorname{FOG}\left(\pi, \pi^{\prime}\right) 11$ In $\operatorname{FOG}\left(\pi, \pi^{\prime}\right)$, we let $P$ be a path from the first vertex of $C$ to the first vertex of $C^{\prime}$, and $P^{\prime}$ be a path from the first vertex of $C^{\prime}$ to the first vertex of $C$. Consider the cycle $C^{\prime \prime}$ obtained by concatenating $P$ and $P^{\prime}$. As a cycle of $\operatorname{FOG}\left(\pi, \pi^{\prime}\right)$, we can map it to a cycle $\pi^{\prime \prime}$ of $\mathcal{R}(\mathcal{G})$ (alternating $\pi$ and $\pi^{\prime}$ depending on the colours of the traversed edges), so that $C^{\prime \prime}$ is a cycle (of length 1 ) of FOG $\left(\pi^{\prime \prime}\right)$. By the divergence of $\mathcal{G}, \pi^{\prime \prime}$ is positive or negative. Suppose for instance that it is positive. Since $(s, r)$ is the first state of both $\pi$ and $\pi^{\prime \prime}$, we can construct the $\operatorname{FOG}\left(\pi, \pi^{\prime \prime}\right)$, in which $C$ is a blue cycle and $C^{\prime \prime}$ is a red cycle, both sharing the same first vertex. We then conclude with the previous case. A similar reasoning with $\pi^{\prime}$ applies to the case that $\pi^{\prime \prime}$ is negative. Therefore, in all cases, we reached a contradiction.

${ }^{11}$ Indeed, one can apply the following construction. We start from a fixed vertex and reach a red simple cycle. We fix a vertex of this red cycle, and from it we can reach a blue simple cycle. We fix a vertex of this blue cycle, and from it we can reach a red simple cycle. There is a finite number of red and blue simple cycles, so we keep alternating between red and blue until we reach a previously seen red simple cycle. This red cycle and, for example, the previous blue one can reach each other. 
To finish the proof of the direct implication of Proposition 5, we suppose that the two simple cycles $\pi$ and $\pi^{\prime}$ in the same SCC of $\mathcal{R}(\mathcal{G})$ do not share any states. By strong connectivity, in $\mathcal{R}(\mathcal{G})$, there exists a path $\pi_{1}$ from the first state of $\pi$ to the first state of $\pi^{\prime}$, and a path $\pi_{2}$ from the first state of $\pi^{\prime}$ to the first state of $\pi$. Consider the cycle of $\mathcal{R}(\mathcal{G})$ obtained by concatenating $\pi_{1}$ and $\pi_{2}$. By divergence of $\mathcal{G}$, it must be positive or negative. Since it shares a state with both $\pi$ and $\pi^{\prime}$, Lemma 2 allows us to prove a contradiction in both cases. This concludes the proof of Proposition 5 .

Value computation. We will now explain how to compute the values of a divergent weighted timed game $\mathcal{G}$. Remember that the function Val maps configurations of $S \times \mathbb{R}_{\geqslant 0}^{X}$ to a value in $\mathbb{R}_{\infty}=\mathbb{R} \cup\{-\infty,+\infty\}$. The semi-algorithm of [9] relies on the same principle as the value iteration algorithm used in the untimed setting, only this time we compute the greatest fixed point of operator $\mathcal{F}: \mathbb{R}_{\infty}^{S \times \mathbb{R}_{\geqslant 0}^{X}} \rightarrow \mathbb{R}_{\infty}^{S \times \mathbb{R}_{\geqslant 0}^{X}}$, defined by $\mathcal{F}(\boldsymbol{x})_{(s, \nu)}=0$ if $s \in S_{t}$, and otherwise

$$
\mathcal{F}(\boldsymbol{x})_{(s, \nu)}= \begin{cases}\sup _{(s, \nu) \stackrel{d, \delta}{\longrightarrow}\left(s^{\prime}, \nu^{\prime}\right)} d \times \text { Weight }(s)+\text { Weight }(\delta)+\boldsymbol{x}_{\left(s^{\prime}, \nu^{\prime}\right)} & \text { if } s \in S_{\mathrm{Max}} \\ \inf _{(s, \nu) \stackrel{d, \delta}{\longrightarrow}\left(s^{\prime}, \nu^{\prime}\right)} d \times \text { Weight }(s)+\text { Weight }(\delta)+\boldsymbol{x}_{\left(s^{\prime}, \nu^{\prime}\right)} & \text { if } s \in S_{\mathrm{Min}}\end{cases}
$$

where $(s, \nu) \stackrel{d, \delta}{\longrightarrow}\left(s^{\prime}, \nu^{\prime}\right)$ ranges over the edges of the infinite weighted game associated with $\mathcal{G}$ (the one defining its semantics). Then, starting from $\boldsymbol{x}^{0}$ mapping every configuration to $+\infty$, we let $\boldsymbol{x}^{i}=\mathcal{F}\left(\boldsymbol{x}^{i-1}\right)$ for all $i>0$. Since $\boldsymbol{x}^{0}$ is piecewise affine (even constant), and $\mathcal{F}$ preserves piecewise affinity, all iterates $\boldsymbol{x}^{i}$ are piecewise affine with a finite amount of pieces. In [1, it is proved that $\boldsymbol{x}^{i}$ has at most a number of pieces linear in the size of $\mathcal{R}(\mathcal{G})$ and exponential in $i 12$

First, we can compute the set of configurations having value $+\infty$. Indeed, the region automaton $\mathcal{R}(\mathcal{G})$ can be seen as a reachability two-player game $\mathcal{S}(\mathcal{G})$ by saying that $(s, r)$ belongs to Min (Max, respectively) if $s \in S_{\text {Min }}\left(s \in S_{\text {Max }}\right.$, respectively). Notice that if $\operatorname{Val}(s, \nu)=+\infty$, then for all $\nu^{\prime} \in[\nu], \operatorname{Val}\left(s, \nu^{\prime}\right)=+\infty$. Therefore, a configuration $(s, \nu)$ cannot reach the target states if and only if $(s,[\nu])$ is not in the attractor of Min to the targets in $\mathcal{S}(\mathcal{G})$. As a consequence, we can compute all such states of $\mathcal{S}(\mathcal{G})$ with complexity linear in the size of $\mathcal{R}(\mathcal{G})$.

We then decompose $\mathcal{R}(\mathcal{G})$ in SCCs. By Proposition $[5$, each SCC is either positive or negative (i.e. it contains only positive cycles, or only negative ones). Then, in order to find the sign of a component, it suffices to find one of its simple cycles, for example with a depth-first search, then compute the weight of one play following it.

As we did for weighted (untimed) games, we then compute values in inverse topological order over the SCCs. Once the values of all configurations in $(s, r)$ appearing in previously considered SCCs have been computed, they are no longer modified in further computation. This is the case, in particular, for all pairs $(s, r)$ that have value $+\infty$, that we precompute from the beginning. In order to resolve a positive SCC of $\mathcal{R}(\mathcal{G})$, we apply $\mathcal{F}$ on the current piecewise affine function, only modifying the pieces appearing in the SCC, until reaching a fixed point over these pieces. In order to resolve a negative SCC of $\mathcal{R}(\mathcal{G})$, we compute the attractor for Max to the previously computed SCCs: outside of this attractor, we set the value to $-\infty$. Then, we apply $\mathcal{F}$ for pieces appearing in the SCC, initialising them to $-\infty$ (equivalently, we compute in the dual game, that is a positive SCC), until reaching a fixed point over these pieces. The next proposition contains the correction and termination arguments that where presented in Propositions 2, 3, and 4 for the untimed setting:

Proposition 6. Let $\mathcal{G}$ be a divergent game with no configurations of value $+\infty$.

1. The value iteration algorithm applied on a positive $S C C$ of $\mathcal{R}(\mathcal{G})$ with $n$ states stabilises after at most $n$ steps.

2. In a negative $S C C$, states $(s, r)$ of $\mathcal{R}(\mathcal{G})$ of value $-\infty$ are all the ones not in the attractor for Max to the targets.

12 For divergent games with only non-negative weights, the fixed point is reached after a number of steps linear in the size of the region automaton [9]: overall, this leads to a doubly exponential complexity. 
3. The value iteration algorithm, initialised with $-\infty$, applied on a negative $S C C$ of $\mathcal{R}(\mathcal{G})$ with $n$ states, and no vertices of value $-\infty$, stabilises after at most $n$ steps.

By the complexity results of [1, Thm. 3], we obtain a doubly exponential time algorithm computing the value of a divergent weighted timed game. This shows that the value problem is in 2-EXPTIME for divergent weighted timed game. The proof for EXPTIME-hardness comes from a reduction of the problem of solving timed games with reachability objectives [20. To a reachability timed game, we simply add weights 1 on every transition and 0 on every state, making it a divergent weighted timed game. Then, Min wins the reachability timed game if and only if the value in the weighted timed game is lower than threshold $\alpha=|S| \times|\operatorname{Reg}(X, M)|$. A complete proof can be found in Appendix D.

In an SCC of $\mathcal{R}(\mathcal{G})$, the value iteration algorithm of [1] allows us to compute an $\varepsilon$-optimal strategy for both players (for configurations having a finite value), that is constant (delay or fire a transition) over each piece of the piecewise affine value function. As in the untimed setting, we may then compose such $\varepsilon$-optimal strategies to obtain an $\varepsilon^{\prime}$-optimal strategy in $\mathcal{G}\left(\varepsilon^{\prime}\right.$ is greater than $\varepsilon$, but can be controlled with respect to the number of SCCs in $\mathcal{R}(\mathcal{G})$ ).

Class decision. Deciding if a weighted timed game is divergent is PSPACE-complete. The complete proof is given in Appendix E, and is an extension of the untimed setting NL-complete result, but this time we reason on regions, hence the exponential blowup in complexity: it heavily relies on Proposition 5, as well as the corner-point abstraction to keep a compact representation of plays.

\section{Conclusion}

In this article, we introduced the first decidable class of weighted timed games with arbitrary weights, with no restrictions on the number of clocks. Future work include the approximation problem for a larger class of weighted timed games (divergent ones where we also allow cycles of weight exactly 0 ), already studied with only non-negative weights by [10].

\section{References}

1. Rajeev Alur, Mikhail Bernadsky, and P. Madhusudan. Optimal reachability for weighted timed games. In Proceedings of the 31st International Colloquium on Automata, Languages and Programming (ICALP'04), volume 3142 of LNCS, pages 122-133. Springer, 2004.

2. Rajeev Alur and David L. Dill. A theory of timed automata. Theoretical Computer Science, 126(2):183-235, 1994.

3. Rajeev Alur, Salvatore La Torre, and George J. Pappas. Optimal paths in weighted timed automata. Theoretical Computer Science, 318(3):297-322, 2004.

4. Eugene Asarin and Oded Maler. As soon as possible: Time optimal control for timed automata. In Hybrid Systems: Computation and Control, volume 1569 of LNCS, pages 19-30. Springer, 1999.

5. Gerd Behrmann, Ansgar Fehnker, Thomas Hune, Kim G. Larsen, Judi Romijn, and Frits W. Vaandrager. Minimum-cost reachability for priced timed automata. In Proceedings of the 4th International Workshop on Hybrid Systems: Computation and Control (HSCC'01), volume 2034 of LNCS, pages 147-161. Springer, 2001.

6. Patricia Bouyer, Thomas Brihaye, Véronique Bruyère, and Jean-François Raskin. On the optimal reachability problem of weighted timed automata. Formal Methods in System Design, 31(2):135-175, 2007.

7. Patricia Bouyer, Thomas Brihaye, and Nicolas Markey. Improved undecidability results on weighted timed automata. Information Processing Letters, 98(5):188-194, 2006.

8. Patricia Bouyer, Ed Brinksma, and Kim G. Larsen. Optimal infinite scheduling for multi-priced timed automata. Formal Methods in System Design, 32(1):3-23, 2008.

9. Patricia Bouyer, Franck Cassez, Emmanuel Fleury, and Kim G. Larsen. Optimal strategies in priced timed game automata. In Proceedings of the 24th Conference on Foundations of Software Technology and Theoretical Computer Science (FSTTCS'04), volume 3328 of LNCS, pages 148-160. Springer, 2004. 
10. Patricia Bouyer, Samy Jaziri, and Nicolas Markey. On the value problem in weighted timed games. In Proceedings of the 26th International Conference on Concurrency Theory (CONCUR'15), volume 42 of Leibniz International Proceedings in Informatics, pages 311-324. Leibniz-Zentrum für Informatik, 2015.

11. Romain Brenguier, Franck Cassez, and Jean-François Raskin. Energy and mean-payoff timed games. In Proceedings of the 17th International Conference on Hybrid Systems: Computation and Control (HSCC'14), pages 283-292. ACM, 2014.

12. Thomas Brihaye, Véronique Bruyère, and Jean-François Raskin. On optimal timed strategies. In Proceedings of the Third international conference on Formal Modeling and Analysis of Timed Systems (FORMATS'05), volume 3829 of LNCS, pages 49-64. Springer, 2005.

13. Thomas Brihaye, Gilles Geeraerts, Axel Haddad, Engel Lefaucheux, and Benjamin Monmege. Simple priced timed games are not that simple. In Proceedings of the 35th IARCS Annual Conference on Foundations of Software Technology and Theoretical Computer Science (FSTTCS'15), volume 45 of LIPIcs, pages 278-292. Schloss Dagstuhl-Leibniz-Zentrum für Informatik, 2015.

14. Thomas Brihaye, Gilles Geeraerts, Axel Haddad, and Benjamin Monmege. To reach or not to reach? Efficient algorithms for total-payoff games. In Proceedings of the 26th International Conference on Concurrency Theory (CONCUR'15), volume 42 of LIPIcs, pages 297-310. Schloss Dagstuhl-LeibnizZentrum für Informatik, 2015.

15. Thomas Brihaye, Gilles Geeraerts, Axel Haddad, and Benjamin Monmege. Pseudopolynomial iterative algorithm to solve total-payoff games and min-cost reachability games. Acta Informatica, 2016.

16. Thomas Brihaye, Gilles Geeraerts, Shankara Narayanan Krishna, Lakshmi Manasa, Benjamin Monmege, and Ashutosh Trivedi. Adding negative prices to priced timed games. In Proceedings of the 25th International Conference on Concurrency Theory (CONCUR'14), volume 8704, pages 560-575. Springer, 2014.

17. Thomas Dueholm Hansen, Rasmus Ibsen-Jensen, and Peter Bro Miltersen. A faster algorithm for solving one-clock priced timed games. In Proceedings of the 24th International Conference on Concurrency Theory (CONCUR'13), volume 8052 of LNCS, pages 531-545. Springer, 2013.

18. Neil Immerman. Number of quantifiers is better than number of tape cells. Journal of Computer and System Sciences, 22(3):384-406, 1981.

19. Neil Immerman. Nondeterministic space is closed under complementation. SIAM Journal on Computing, 17:935-938, 1988.

20. Marcin Jurdziński and Ashutosh Trivedi. Reachability-time games on timed automata. In Proceedings of the 34th International Colloquium on Automata, Languages and Programming (ICALP'O7), volume 4596 of $L N C S$, pages 838-849. Springer, 2007.

21. Leonid Khachiyan, Endre Boros, Konrad Borys, Khaled Elbassioni, Vladimir Gurvich, Gabor Rudolf, and Jihui Zhao. On short paths interdiction problems: Total and node-wise limited interdiction. Theory of Computing Systems, 43(2):204-233, 2008.

22. Anuj Puri. Dynamical properties of timed automata. Discrete Event Dynamic Systems, 10(1-2):87$113,2000$.

23. Michał Rutkowski. Two-player reachability-price games on single-clock timed automata. In Proceedings of the Ninth Workshop on Quantitative Aspects of Programming Languages (QAPL'11), volume 57 of EPTCS, pages 31-46, 2011.

24. Walter J. Savitch. Relationships between nondeterministic and deterministic tape complexities. Journal of Computer and System Sciences, 4(2):177-192, 1970.

25. Róbert Szelepcsényi. The method of forced enumeration for nondeterministic automata. Acta Informatica, 26(3):279-284, 1988. 


\section{A Technical lemmas regarding value iteration algorithms}

Lemma 3 (untimed setting notations). For all $i<j \in \mathbb{N}$, if $\boldsymbol{x}^{j} \neq \boldsymbol{x}^{i}$ then for all $v \in V$ there exists $v^{\prime}$ and a play $\rho$ from $v$ to $v^{\prime}$ with $|\rho|=j-i$ and Weight $\left.(\rho)=\boldsymbol{x}_{v}^{j}-\boldsymbol{x}_{v^{\prime}}^{i}\right)$.

Proof. Let us fix $i$, and prove it by induction on $j>i$.

Initialisation : If $j=i+1$, we applied one step of the value iteration algorithm between $\boldsymbol{x}^{i}$ and $\boldsymbol{x}^{j}$, so for all $v$ there exists $v^{\prime}$ and an edge $e=v \rightarrow v^{\prime}$ such that Weight $(e)=\boldsymbol{x}_{v}^{i+1}-\boldsymbol{x}_{v^{\prime}}^{i}$.

Iteration: We assume the property holds for $j-1>i$, and $\boldsymbol{x}^{j} \neq \boldsymbol{x}^{i}$. We applied one step of the value iteration algorithm between $\boldsymbol{x}^{j-1}$ and $\boldsymbol{x}^{j}$, so for all $v$ there exists $v^{\prime}$ and an edge $e=v \rightarrow v^{\prime}$ such that Weight $(e)=\boldsymbol{x}_{v}^{j}-\boldsymbol{x}_{v^{\prime}}^{j-1}$. We apply the property on $i$ and $j-1\left(\boldsymbol{x}^{j-1} \neq \boldsymbol{x}^{i}\right.$ because $\boldsymbol{x}^{j} \neq \boldsymbol{x}^{i}$ and as soon as $\boldsymbol{x}$ stabilises, the fixed point is reached and the iteration stops), and obtain that for all $v^{\prime} \in V$ there exists $v^{\prime \prime}$ and a play $\rho$ from $v^{\prime}$ to $v^{\prime \prime}$ with $|\rho|=j-1-i$ and Weight $(\rho)=\boldsymbol{x}_{v^{\prime}}^{j-1}-\boldsymbol{x}_{v^{\prime \prime}}^{i}$. Then we define $\rho^{\prime}=v \rightarrow v^{\prime} \stackrel{\rho}{\rightarrow} v^{\prime \prime}$ and it holds that $\left|\rho^{\prime}\right|=j-i$ and Weight $\left(\rho^{\prime}\right)=\boldsymbol{x}_{v}^{j}-\boldsymbol{x}_{v^{\prime \prime}}^{i}$.

Lemma 4 (timed setting notations). For all $i<j \in \mathbb{N}$, if $\boldsymbol{x}^{j} \neq \boldsymbol{x}^{i}$ then, for all configurations $(s, \nu)$, there exists $\left(s^{\prime}, \nu^{\prime}\right)$ and a play $\rho$ from $(s, \nu)$ to $\left(s^{\prime}, \nu^{\prime}\right)$ with $|\rho|=j-i$ and $\operatorname{Weight}(\rho)=$ $\boldsymbol{x}_{(s, \nu)}^{j}-\boldsymbol{x}_{\left(s^{\prime}, \nu^{\prime}\right)}^{i}$.

Proof. Let us fix $i$, and prove it by induction on $j>i$.

Initialisation: If $j=i+1$, we applied $\mathcal{F}$ once between $\boldsymbol{x}^{i}$ and $\boldsymbol{x}^{j}$, so for all configurations $(s, \nu)$ there exists $\left(s^{\prime}, \nu^{\prime}\right)$ and a transition $(s, \nu) \stackrel{d, \delta}{\longrightarrow}\left(s^{\prime}, \nu^{\prime}\right)$ of weight $\boldsymbol{x}_{(s, \nu)}^{j}-\boldsymbol{x}_{\left(s^{\prime}, \nu^{\prime}\right)}^{i}$.

Iteration: We assume the property holds for $j-1>i$, and $\boldsymbol{x}^{j} \neq \boldsymbol{x}^{i}$. We applied $\mathcal{F}$ once between $\boldsymbol{x}^{j-1}$ and $\boldsymbol{x}^{j}$, so for all configurations $(s, \nu)$, there exists $\left(s^{\prime}, \nu^{\prime}\right)$ and a transition $(s, \nu) \stackrel{d, \delta}{\longrightarrow}\left(s^{\prime}, \nu^{\prime}\right)$ of weight $\boldsymbol{x}_{(s, \nu)}^{j}-\boldsymbol{x}_{\left(s^{\prime}, \nu^{\prime}\right)}^{i}$. We apply the property on $i$ and $j-1\left(\boldsymbol{x}^{j-1} \neq \boldsymbol{x}^{i}\right.$ because $\boldsymbol{x}^{j} \neq \boldsymbol{x}^{i}$ and as soon as $\boldsymbol{x}$ stabilises, the fixed point is reached and the iteration stops), and obtain that for all configurations $\left(s^{\prime}, \nu^{\prime}\right)$, there exists $\left(s^{\prime \prime}, \nu^{\prime \prime}\right)$ and a play $\rho$ from $\left(s^{\prime}, \nu^{\prime}\right)$ to $\left(s^{\prime \prime}, \nu^{\prime \prime}\right)$ with $|\rho|=j-1-i$ and Weight $\left.(\rho)=\boldsymbol{x}_{\left(s^{\prime}, \nu^{\prime}\right)}^{j-1}-\boldsymbol{x}_{\left(s^{\prime \prime}, \nu^{\prime \prime}\right)}^{i}\right)$. Then we define $\rho^{\prime}=(s, \nu) \stackrel{d, \delta}{\longrightarrow}\left(s^{\prime}, \nu^{\prime}\right) \stackrel{\rho}{\rightarrow}\left(s^{\prime \prime}, \nu^{\prime \prime}\right)$ and it holds that $\left|\rho^{\prime}\right|=j-i$ and Weight $\left(\rho^{\prime}\right)=\boldsymbol{x}_{(s, \nu)}^{j}-\boldsymbol{x}_{\left(s^{\prime \prime}, \nu^{\prime \prime}\right)}^{i}$.

\section{B FOG and corner-point abstraction}

If $\nu$ is a valuation and $\varepsilon>0, \mathcal{B}_{\infty}(\nu, \varepsilon)$ denotes the open ball of radius $\varepsilon$ centered into $\nu$ for the infinity norm $\|\cdot\|_{\infty}$ over $\mathbb{R}_{\geqslant 0}^{X}$ : $\left\|\nu-\nu^{\prime}\right\|_{\infty}=\max _{x \in X}\left|\nu(x)-\nu^{\prime}(x)\right|$. We let $W$ be the biggest weight appearing in $\mathcal{G}$ in absolute value. If $\rho$ and $\bar{\rho}$ are plays in $\mathcal{G}$ following respectively a play $\pi$ and its copy in $\overline{\mathcal{G}}$, we denote by $d(\rho, \bar{\rho})$ the distance between those two plays, defined as the sum of the differences in absolute value between the delays on the edges of $\rho$ and $\bar{\rho}$. By triangular inequality, we obtain $\mid$ Weight $(\rho)-$ Weight $(\bar{\rho}) \mid \leqslant W d(\rho, \bar{\rho})$, since the same transitions are fired in $\rho$ and $\bar{\rho}$, with only different delays. We will now relate an edge $v \stackrel{\bar{\rho}}{\rightarrow} v^{\prime}$ of a FOG with an actual play $\rho$ in the original timed game, while controlling the distance between Weight $(\rho)$ and Weight $(\bar{\rho})$ :

Lemma 5. Let $\pi$ be a cycle of $\mathcal{R}(\mathcal{G})$, and $v \stackrel{\bar{\rho}}{\rightarrow} v^{\prime}$ be an edge of $\operatorname{FOG}(\pi)$. For all $\varepsilon>0$ and $\nu \in r \cap \mathcal{B}_{\infty}(v, \varepsilon)$, there exists $\nu^{\prime} \in r \cap \mathcal{B}_{\infty}\left(v^{\prime}, \varepsilon\right)$ and $\rho$ play in $\mathcal{G}$ from $\nu$ to $\nu^{\prime}$ following $\pi$ such that $\mid$ Weight $(\rho)-$ Weight $(\bar{\rho})|\leqslant 2 \varepsilon| \pi \mid W$.

Proof. By the previous explanation, it is sufficient to find a play $\rho$ such that $d(\rho, \bar{\rho}) \leqslant 2 \varepsilon|\pi|$. By induction, it is sufficient to prove a similar result only for a single edge $(s, r) \stackrel{\delta=\left(s, g, Y, s^{\prime}\right)}{\longrightarrow}\left(s^{\prime}, r^{\prime}\right)$ of the region automaton $\mathcal{R}(\mathcal{G})$, between regions $r$ and $r^{\prime}$. We thus consider a play $(s, v) \stackrel{d, \delta}{\longrightarrow}\left(s^{\prime}, v^{\prime}\right)$ in the closed timed game $\overline{\mathcal{G}}$ from a corner $v \in \bar{r}$ to a corner $v^{\prime} \in \overline{r^{\prime}}$. Consider a valuation $\nu \in$ $r \cap \mathcal{B}_{\infty}(v, \varepsilon)$. We now explain how to construct a valuation $\nu^{\prime} \in r^{\prime} \cap \mathcal{B}_{\infty}\left(v^{\prime}, \varepsilon\right)$ and $d^{\prime} \geqslant 0$ such that $\nu \stackrel{d^{\prime}, \delta}{\longrightarrow} \nu^{\prime}$ is a valid play in $\mathcal{G}$ and $\left|d-d^{\prime}\right| \leqslant 2 \varepsilon$, which implies the lemma. 
Let $r^{\prime \prime}$ be the time successor region of $r$ such that $r^{\prime \prime}[Y \leftarrow 0]=r^{\prime}$. We let $v^{\prime \prime}=v+d$ be the valuation of $\overline{r^{\prime \prime}}$, just before the possible resets of the clocks of $Y$ in $\overline{\mathcal{G}}: v^{\prime}=v^{\prime \prime}[Y \leftarrow 0]$. Then, the timed successors of $\nu$, i.e. the affine line $\nu+(1,1, \ldots, 1) \mathbb{R}$, intersect the set $r^{\prime \prime} \cap \mathcal{B}_{\infty}\left(v^{\prime \prime}, \varepsilon\right)$ in a valuation $\nu^{\prime \prime}$ : indeed, lines obtained by time elapsing starting from $\nu$ and $v$ are parallel, and $r^{\prime \prime}$ is a time successor of $r$. There exists $d^{\prime}$ such that $\nu^{\prime \prime}=\nu+d^{\prime}$. Moreover, $d^{\prime}=\left\|\nu-\nu^{\prime \prime}\right\|_{\infty} \leqslant\|\nu-v\|_{\infty}+$ $\left\|v-v^{\prime \prime}\right\|_{\infty}+\left\|v^{\prime \prime}-\nu^{\prime \prime}\right\|_{\infty} \leqslant 2 \varepsilon+d$, and $d=\left\|v-v^{\prime}\right\|_{\infty} \leqslant\|v-\nu\|_{\infty}+\left\|\nu-\nu^{\prime}\right\|_{\infty}+\left\|\nu^{\prime}-v^{\prime}\right\|_{\infty} \leqslant 2 \varepsilon+d^{\prime}$ so that $\left|d-d^{\prime}\right| \leqslant 2 \varepsilon$. Letting $\nu^{\prime}=\nu^{\prime \prime}[Y \leftarrow 0]$, we have $\nu \stackrel{d^{\prime}, \delta}{\longrightarrow} \nu^{\prime}$ and $\nu^{\prime} \in r^{\prime} \cap \mathcal{B}_{\infty}\left(v^{\prime}, \varepsilon\right)$.

This lemma is stronger than Lemma 1, and thus proves it.

\section{Proofs of correctness and termination of the algorithm for divergent weighted timed games}

Proof (of Proposition [6]1). Let $W=\max _{a \in \Delta \cup S} \mid$ Weight $(a) \mid$ be the greatest weight in the game. There are no negative cycles in the SCC, therefore there are no configurations with value $-\infty$, and all values are finite. Let $K$ be a bound on the values $\left|\boldsymbol{x}_{(s, \nu)}^{n}\right|$ obtained after $n$ steps of the algorithm 13 Let us fix an integer $p>\left(2 K+(n-1)^{2}(W+M W)\right) n$. We will show that the values obtained after $n+p$ steps are identical to those obtained after $n$ steps only. Therefore, since the algorithm computes non-increasing sequences of values, we have indeed stabilised after $n$ steps only. Let us assume the existence of a configuration $(s, \nu)$ such that $\boldsymbol{x}_{(s, \nu)}^{n+p}<\boldsymbol{x}_{(s, \nu)}^{n}$. By induction on $p$, we can show (see Lemma 4 in Appendix $\mathrm{A}$ for a detailed proof) the existence of a configuration $\left(s^{\prime}, \nu^{\prime}\right)$ and a finite play $\rho$ from $(s, \nu)$ to $\left(s^{\prime}, \nu^{\prime}\right)$, with length $p$ and weight $\boldsymbol{x}_{(s, \nu)}^{n+p}-\boldsymbol{x}_{\left(s^{\prime}, \nu^{\prime}\right)}^{n}$ : the play is composed of the delays and transitions that optimise successively the min/max operator in $\mathcal{F}$. This finite play being of length greater than $\left(2 K+(n-1)^{2}(W+M W)\right) n$, if we associate each visited configuration $(s, \nu)$ to the state $(s,[\nu])$ of $\mathcal{R}(\mathcal{G})$, there is at least one state of $\mathcal{R}(\mathcal{G})$ appearing more than $2 K+(n-1)^{2}(W+M W)$ times. Thus, it can be decomposed into at least $2 K+(n-1)^{2}(W+M W)$ plays following cycles of $\mathcal{R}(\mathcal{G})$ and at most $(n-1)$ finite plays $\rho_{i}^{\prime}$ visiting each state of $\mathcal{R}(\mathcal{G})$ at most once. All cycles of the SCC being positive, the weight of $\rho$ is at least $\left(2 K+(n-1)^{2}(W+M W)\right)-(n-1)^{2}(W+M W)=2 K$, bounding from below each $\rho_{i}^{\prime}$ 's weight by $-(n-1)(W+M W)$. Then, $\boldsymbol{x}_{(s, \nu)}^{n+p}-\boldsymbol{x}_{\left(s^{\prime}, \nu^{\prime}\right)}^{n} \geqslant 2 K$, so $\boldsymbol{x}_{(s, \nu)}^{n+p} \geqslant 2 K+\boldsymbol{x}_{\left(s^{\prime}, \nu^{\prime}\right)}^{n} \geqslant 2 K-K \geqslant K$. But $K \geqslant \boldsymbol{x}_{(s, \nu)}^{n}$, so $\boldsymbol{x}_{(s, \nu)}^{n+p} \geqslant \boldsymbol{x}_{(s, \nu)}^{n}$, and that is a contradiction.

Much like in the untimed setting, negative SCCs can be resolved using a dual method. First, we characterise the $-\infty$ values as regions of $\mathcal{S}(\mathcal{G})$ where Max can not unilaterally guarantee to reach the targets.

Proof (of Proposition [6 [2). Consider a state $(s, r)$ of $\mathcal{S}(\mathcal{G})$ in the attractor for Max to the targets. Then, if Max applies a winning memoryless strategy for the reachability objective to the target states, for all $\nu \in r$, all strategies of Min will generate a play from $(s, \nu)$ reaching a target after at most $|\mathcal{S}(\mathcal{G})|$ steps. This implies that $(s, \nu)$ has a finite (lower) value in the game.

Reciprocally, if $(s, r)$ is not in the attractor, by determinacy of timed games with reachability objectives, for all $\nu \in r$, Min has a (memoryless) strategy $\sigma_{\text {Min }}$ to ensure that no strategy of Max permits to reach a target state from $(s, \nu)$. Applying $\sigma_{\text {Min }}$ long enough to generate a play following many negative cycles, before switching to a strategy allowing Min to reach the target (such a strategy exists since no configuration has value $+\infty$ in the game), allows Min to obtain from $(s, \nu)$ a negative weight as small as possible. Thus, $(s, \nu)$ has value $-\infty$.

Thus, given a negative SCC, we can compute configurations of value $-\infty$ in time polynomial in the SCC's size. Then, finite values of other configurations can be computed by applying $\mathcal{F}$.

\footnotetext{
${ }^{13}$ The value iteration emulates the attractor computation, so every value is finite after $n$ steps. Moreover, functions $(s, \nu) \mapsto \boldsymbol{x}_{(s, \nu)}^{n}$ are piecewise affine with a finite number of pieces over a compact space, allowing us to obtain this uniform bound $K$.
} 
Proof (of Proposition [6 3). From a negative SCC $\mathcal{S}(\mathcal{G})$ that has no more configuration of value $+\infty$ or $-\infty$, consider the dual (positive) SCC $\widetilde{\mathcal{G}}$ obtained by: $(i)$ switching states of Min and Max; (ii) taking the opposite of every weight in states and transitions. Sets of strategies of both players are exchanged in those two games, so that the upper value in $\mathcal{G}$ is equal to the opposite of the lower value in $\widetilde{\mathcal{G}}$, and vice versa. Since weighted games are determined, the value of $\mathcal{G}$ is the opposite of the value of $\widetilde{\mathcal{G}}$. Then, the value of $\mathcal{G}$ can be deduced from the value of $\widetilde{\mathcal{G}}$, for which Proposition 6] [1] applies.

It is then immediate that the values computed with this computation of the smallest fixed point of $\mathcal{F}$ are exactly the opposite values of the ones computed in the dual positive SCC.

\section{EXPTIME-hardness of the value problem in divergent weighted timed games}

Let us show EXPTIME-hardness of the value problem on divergent weighted timed games by reducing to reachability in a timed game 20. Consider an instance of timed game reachability with a timed game $\mathcal{A}$ with target states and a configuration $(s, \nu)$. Let us call Min the player trying to enforce reachability of a target state in $\mathcal{A}$ and Max the one opposing it. We construct from $\mathcal{A}$ a weighted timed game $\mathcal{G}$ by considering the same game with all states weights at 0 and all transition weights at 1 . Notice that $\mathcal{G}$ is divergent. Let us define $\alpha$ as an upper bound on the number of states in the region automaton of $\mathcal{A}$, using classical bounds on the number of regions: $\alpha=|S|(4(M+1))^{|X|}|X| !(M$ is the upper bound on clock values). We consider the instance of the value problem defined with $\mathcal{G}$, the configuration $(s, \nu)$, and the bound $\alpha$. Then $\operatorname{Val}_{\mathcal{G}}(s, \nu) \leqslant \alpha$ if and only if $(s, \nu)$ can reach the target vertices in $\mathcal{A}$.

One direction of this statement's proof is direct by definition of having a value smaller than $+\infty$, and the other comes from the fact that reachability in $\mathcal{A}$ implies reachability in the region game of $\mathcal{A}$ in less than $\alpha$ transitions, and that implies reachability in $\mathcal{G}$ with weight below $\alpha$ from the configuration $(s, \nu)$ to a target state, therefore $\operatorname{Val}_{\mathcal{G}}(s, \nu) \leqslant \alpha$.

Notice that this reduction is polynomial in the size of $\mathcal{A}$ because the bound $\alpha$ can be encoded and computed in binary.

\section{E Deciding divergence for weighted timed games}

Let us show how to decide if a game is not divergent. By Proposition 5, it suffices to search for an SCC of the region automaton containing a non-negative simple cycle (i.e. there exists a play following it of weight in $(-1,+\infty))$ and a non-positive one (i.e. there exists a play following it of weight in $(-\infty, 1))$. Since simple cycles have a length bounded by $\alpha=|S| \times|\operatorname{Reg}(X, M)|=$ $|S|(4(M+1))^{|X|}|X|$ !, we simply need to test the existence of two cycles of length at most $\alpha$, in the same SCC, one being non-negative and the other non-positive: notice that this condition indeed implies the non-divergence by the same proof as in Proposition 5. Finally, notice that, by Lemma 1, given a cycle of the region automaton, we can decide if there exists a play following it with weight in $(-\infty, 1)$ (respectively, $(-1,+\infty)$ ), by guessing a play following the corners in $\overline{\mathcal{G}}$ (game where strict inequalities in guards are replaced with non-strict ones) and checking that its accumulated weight is an integer in $(\infty, 0]$ (respectively, $[0,+\infty)$ ). We test this condition in NPSPACE, by the same techniques as in the untimed setting: we guess a starting region, and a starting corner, for both cycles, we check in polynomial space that the regions are in the same SCC of $\mathcal{R}(\mathcal{G})$, and we guess on-the-fly the two cycles, i.e. the sequences of regions with one of their corners, keeping in memory their accumulated weight, stopping when we found two cycles of $\mathcal{R}(\mathcal{G})$, or if their length becomes larger than $\alpha$. Note the accumulated weights are integers bounded (in absolute value) by $\alpha \times \max _{a \in \Delta \cup S} \mid$ Weight $(a) \mid$, and can thus be stored in polynomial space. This shows that deciding the divergence is in coNPSPACE $=$ NPSPACE $=$ PSPACE (using the theorems of Immerman-Szelepcsényi [19]25] and Savitch [24]).

Let us now show the PSPACE-hardness (indeed the coPSPACE, which is identical) by a reduction from the reachability problem in a timed automaton. As in the untimed setting, we consider 
a timed automaton with a starting state and a different target state without outgoing transitions. We construct from it a weighted timed game by distributing all states to Min, and equipping all transitions with weight 1 , and all states with weight 0 . We also add a loop with weight -1 on the target state, and a transition from the target state to the initial state with weight 0 , both resetting all transitions and bounded by a delay of 1 . Then, the weighted timed game is not divergent if and only if the target can be reached from the initial state in the timed automaton. 